\title{
Comunicación y Marcos de Acción Colectiva en el Movimiento Global de Ecoaldeas
}

\author{
Pereira Salazar, Claudio ${ }^{1}$; Bernete García, Francisco ${ }^{2}$
}

Recibido: 6 de mayo de 2017 / Aceptado: 29 de mayo de 2017

Resumen. En el presente trabajo se investigó sobre los procesos de comunicación y los marcos de acción colectiva en el Movimiento Global de Ecoaldeas. A partir de la teoría de la Mediación Social se analizaron (1) los sitios web, (2) documentos elaborados en el movimiento y (3) entrevistas a los participantes. Los resultados dan cuenta de un movimiento global contemporáneo que, apoyado por internet, construye un proyecto global de cambio social, genera identidad colectiva y promueve una acción social transformadora. Un movimiento que aprovecha las ventajas las Tecnologías de la información y la comunicación como medio de comunicación y expresión de sus reivindicaciones.

Palabras clave: Comunicación; cambio social; movimientos sociales; marcos de acción colectiva; ecoaldeas.

\section{[en] Communication and Collective Action Frames in Global Ecovillage Network}

Abstract. In the present work we investigated the communication processes and collective action frameworks in the Global Ecovillage Movement. From the Social Mediation theory we analyzed (1) the websites, (2) documents elaborated in the movement and (3) interviews with the participants. The results show a contemporary global movement that, supported by the Internet, builds a global project of social change, generates collective identity and promotes transformative social action. A movement that takes advantage of the technologies of information and communication as a means of communication and expression of their demands.

Keywords: communication; social change; social movements; collective action frames; ecovillage.

Sumario. Introducción. 2. Fundamentos. 3. Método. 4. Resultados. 5. Conclusiones. 6. Referencias bibliográfícas.

Cómo citar: Pereira Salazar, Claudio; Bernete García, Francisco (2017) "Comunicación y Marcos de Acción Colectiva en el Movimiento Global de Ecoaldea”, en Mediaciones Sociales 16, 11-26

1 Universidad de Antofagasta (Chile).

claudio.pereira@uantof.cl

2 Universidad Complutense de Madrid (España).

fbernete@ucm.es 


\section{Introducción}

Lo que hoy entendemos por civilización moderna es la materialización objetiva de lo que los especialistas en sistemas y organizaciones denominarían "complejidad creciente". En esta complejidad participan diversos colectivos y movimientos sociales de variados intereses y organizaciones. Algunos de estos movimientos sociales, gracias al uso de las nuevas tecnologías, tienen la posibilidad de "globalizarse", traspasando barreras territoriales y transnacionalizando sus visiones de mundo.

El Movimiento Global de Ecoaldeas es un movimiento global contemporáneo orientado a la construcción de propuestas para el desarrollo humano sostenible y expresión de una acción social transformadora, que promueve espacios de movilización a nivel planetario y particularidades en sus repertorios de movilización a nivel local.

El objetivo general de esta investigación fue el estudio de los marcos de acción colectiva contenidos en las representaciones que el Movimiento Global de Ecoaldeas ofrece sobre sí mismo y su entorno en sus sitios web. Se buscó profundizar el análisis del movimiento global en seis dimensiones:

1. la comunicación y el uso de internet en el movimiento.

2. la participación y la acción colectiva.

3. la visión del mundo, criterios de caracterización y procesos identitarios de los participantes del movimiento.

4. la organización y el funcionamiento de los proyectos del movimiento.

5. las redes que constituyen el movimiento, la relación con el entorno y su forma de coordinación.

6. los procesos de marcos de acción colectiva.

\section{Fundamentos}

Los fundamentos de la investigación parten de las perspectivas contemporáneas para el estudio de los movimientos sociales, centradas en los aspectos simbólicos que fundamentan los marcos culturales sobre los que se basa la acción colectiva y la construcción de identidad. La formulación de los objetivos se basó en la revisión teórica de los estudios sobre movimientos sociales, como los procesos de identidad (Laraña \& Gusfield, 1994), acción colectiva (Tarrow, 1997), marcos culturales de movilización (Snow \& Benford, 1988), repertorios de acción (Labé, 2011), globalización de los movimientos sociales (Maldonado, 2008) y las mediaciones tecnológicas que favorecen la emergencia de los nuevos movimientos globales (Ortiz, 2008).

El término "marcos de acción colectiva" procede de la propuesta de Snow y Benford en el desarrollo del concepto de "framing" (creación de marcos de referencia), usado para conceptualizar el "trabajo de significación" (signifying work) que realizan los movimientos sociales. Según los autores, "los movimientos 
funcionan como portadores y transmisores de creencias e ideas movilizantes, pero también están activamente comprometidos en la producción de significado para participantes, antagonistas y observadores (...) los movimientos pueden así ser interpretados en parte como agentes de significación (...) ellos enmarcan, o asignan significado e interpretan, sucesos y condiciones pertinentes, cuyo sentido está destinado a movilizar a potenciales seguidores y miembros, a fomentar apoyo entre los espectadores y a desmovilizar a los antagonistas" (Snow y Benford, 1988). Entonces, los marcos de referencia de la acción colectiva, no sólo destacan ciertos aspectos de la realidad, sino que también actúan como base para la atribución y articulación de significados (Hunt, Benfort y Snow, 1994).

El presente artículo se centra en el análisis de la comunicación y los procesos de marcos de acción colectiva. Ambos procesos fundamentales en las propuestas educativas y de coordinación en red.

De acuerdo a Callé (2003), en la actualidad estamos frente a una nueva cultura de acción colectiva que se expresa en la emergencia de los Nuevos Movimientos Globales, una de cuyas características es su reproducción global. La denominada globalización contribuye a hacer del planeta una unidad cultural, temporal y espacial de referencia para el desarrollo de estos movimientos. La globalización tecnológica y mediática propicia la conexión de espacios de descontento y, como en las grandes cumbres oficiales, se genera la posibilidad de unir discursos y formas de acción a escala planetaria. Otra característica de estos movimientos es que se encuentran presididos por identidades abiertas y difusas, conectan lo público y lo privado, lo local y lo global, generando discursos en red, presentando altos grados de conectividad y heterogeneidad favorecido por el uso de las nuevas TIC como es el caso de Internet (Calle, 2007).

El uso generalizado de Internet, como medio de información y expresión de parte de los movimientos sociales contemporáneos, justifica la elección de los sitios web como objeto material de estudio. Los sitios web son un recurso que permite a las organizaciones y redes sociales ganar mayor visibilidad pública para comunicar y dar a conocer sus planteamientos y acciones. Se constituyen en productos comunicativos a través de los cuales podemos acceder a los modelos de representación del mundo que determinado grupo cultural ha puesto a disposición de todos en el ciberespacio. En esos productos comunicativos se reflejan las decisiones tomadas por los miembros de las organizaciones de los movimientos sociales para dar a conocer ciertos aspectos del colectivo y en sus contenidos se hace referencia a la identidad y los marcos de significados que permiten su existencia.

El Movimiento Global de Ecoaldeas es un ejemplo de estos nuevos movimientos globales. Se seleccionó por ser uno de los movimientos sociales contemporáneos que, a pesar de no recibir la atención de los medios tradicionales de comunicación pública, presentan un rápido crecimiento en todo el planeta, experimentando modelos concretos de cambio social. Se distingue de otros movimientos en que su acción movilizadora está centrada en la educación y la práctica de un sistema de vida comunitario, más que en la protesta o en la lucha. 


\section{Método}

El análisis se lleva a cabo con el enfoque teórico metodológico propio de la teoría de la mediación social planteada por el profesor Martín Serrano (2008). En su planteamiento teórico, se entiende por "productos comunicativos" aquellos que han sido fabricados para proveer de información pública a una comunidad, es decir, "un objeto fabricado que tiene un valor de uso concreto: poner la información que han elaborado unos sujetos sociales a disposición de otros" (Martín Serrano, 2004). En este caso, en los sitios web del movimiento se encuentran contenidas las representaciones que dan cuenta de los aspectos identitarios y la actividad que realizan las organizaciones del colectivo social. Basándose en la Teoría de la Mediación desarrollada por Martín Serrano, en los procesos como en los productos y discursos comunicativos de los movimientos sociales podría reconocerse una mediación cognitiva (que afecta al cambio o reproducción de las representaciones individuales y colectivas) y una mediación estructural, que afecta a las formas que finalmente adquieren los productos comunicativos y sus usos sociales (Martín Serrano, 2004).

\subsection{Técnicas y Fuentes de Información}

Como estrategia metodológica se desarrolló el análisis de contenido de los sitios web, a partir de la construcción de un protocolo de análisis. Para algunos contenidos se implementó un análisis de las relaciones lógicas que mantienen entre sí los elementos narrativos que aparecen en los sitios web, como instrumento que permite objetivar la estructura de los modelos comunicativos existentes en los sitios web. Se interpretaron los resultados complementando el análisis con los contenidos de documentos elaborados en el movimiento y entrevistas a los participantes.

Se tomó como universo de estudio la totalidad de sitios web en español, lo que comprende las organizaciones del movimiento presentes en la mayoría de países de América Latina y España. En total se analizaron 77 sitios web. Se consideraron los sitios web en español como un segmento del movimiento global, compartido por la comunidad lingüística de lengua castellana.

Tomando en cuenta los objetivos de la investigación, los focos de interés de los estudios de los movimientos sociales y la exploración detallada de cada uno de los sitios web del universo de estudio, se realizó un listado con los temas referidos al modo en que las organizaciones del movimiento ofrecen representaciones sobre sí mismas y su entorno.

Los sitios web se construyen para dar a conocer la existencia de la organización que representa y promover sus actividades. Los objetos de referencia que interesan para este estudio son los utilizados para identificar y describir ámbitos del "sí mismo" y del "entorno" del movimiento.

- En el ámbito del "sí mismo" se consideran las referencias a: las organizaciones, los participantes y el movimiento global. 
- En el ámbito del entorno: el medio ambiente, el orden social, la educación, la salud, la economía, la espiritualidad.

El conjunto de los datos observados se agrupó en bloques de la siguiente manera:

1. Características de los sitios web: en este bloque se agrupan aquellas categorías que ofrecen información identificativa de los sitios web, sus características y los recursos comunicativos utilizados en su construcción.

2. Aspectos formales de la organización: se agrupan datos sobre la localización de las organizaciones, año de inicio, forma legal, tipo de ecoaldea, recursos materiales de que dispone la organización y áreas de los proyectos en que trabajan.

3. Participación en el movimiento: referencias con que las organizaciones del movimiento representan aspectos de la participación. Proporciona información sobre el número de participantes en la organización, las formas y posibilidades de participación, las formas de reclutamiento y las motivaciones para la participación.

4. Actividades de la organización: información que nos permite explorar las referencias sobre las actividades que realizan las organizaciones del Movimiento Global de Ecoaldeas para su funcionamiento y existencia. En los sitios es posible encontrar referencias sobre diferentes ámbitos de actividades. Se registra información sobre las actividades internas de la organización, las actividades de financiación, las formas de acción social, las actividades educativas y recreativas a las que se hace referencia en los textos.

5. Aspectos organizacionales: la estructura y cultura organizacional tal como aparece en los sitios web. Información que responde a la pregunta sobre cómo funcionan las organizaciones del movimiento y su relación con otras variables. Se recoge información sobre el sistema de liderazgo empleado por las organizaciones, la estructura organizacional, el sistema de toma de decisiones y el sistema de resolución de conflicto.

6. Relaciones de la organización con el entorno: contenidos que representan el tipo de proyectos a través de los cuales el movimiento interviene en su entorno, los vínculos que establece con otras organizaciones y redes, así como el tipo de medios de comunicación utilizados por las organizaciones del movimiento para dar a conocer sus actividades.

7. Criterios de identificación social: bloque compuesto por una serie de variables que codifican contenidos que hacen referencia a la identidad del movimiento en relación a los siguientes ámbitos: identidad principal de la organización, identidad territorial, sistemas de pensamientos con los que se identifica la organización, autodefinición de la organización, identificación con otros movimientos, autodefinición de los participantes, fines de la 
organización, valores y principios que sustentan y definen la acción social del movimiento.

8. Concepción del Mundo: con este último bloque se exploran las representaciones del entorno del movimiento, las formas de interpretar diferentes aspectos de la realidad y sus relaciones con otras variables. Se recoge información sobre los siguientes aspectos: percepción de la sociedad global, críticas al orden social dominante, propuestas de cambio social, concepciones sobre el medio ambiente, la educación, la salud, la economía y la espiritualidad. Todas estas dimensiones permiten abordar los significados que comparten los participantes del movimiento al representar el mundo y las formas de actuar en él.

La base de datos está constituida por 77 registros correspondiente a los sitios web analizados. En los sitios web se realizó la búsqueda de información sobre las 49 variables que constituyen el protocolo de análisis. El software permite organizar la información en diferentes presentaciones de acuerdo a la información que se necesite obtener.

Para el análisis de la información que arrojó la aplicación del protocolo se propuso un diseño basado en la integración de tres niveles de análisis sucesivos.

1. En el primer nivel se analizan los resultados de la distribución de frecuencias de aparición de las categorías.

2. En un segundo nivel de análisis se propuso aplicar en algunos casos el análisis de relaciones lógicas de acuerdo a un plan de explotación definido para algunas de las variables, con el fin de arrojar luz sobre la estructura de los modelos comunicativos y las relaciones entre sus categorías.

3. El tercer nivel de análisis supone una integración de los dos niveles anteriores, articulando los resultados del análisis en ambos niveles con la reflexión teórica y la información contenida en entrevistas, observación participante y otros documentos generados por las organizaciones. Se sintetizaron los resultados más importantes siguiendo como eje conductor las secciones temáticas del estudio.

\section{Resultados}

Abordamos el análisis de los marcos de acción colectiva considerando la comunicación, la identidad, la acción social, las formas de organización, las redes y las propuestas promovidas en el movimiento. El análisis llevado a cabo nos ha permitido conocer mejor los marcos de acción colectiva contenidos en las representaciones que los participantes del Movimiento Global de Ecoaldeas ofrecen sobre sí mismos y su entorno en sus sitios web. A continuación destacamos los resultados mas relevantes del análisis y su valoración: 


\subsection{El uso de internet y la comunicación en el Movimiento Global de Ecoaldeas: reclutamiento, espacio de colaboración y vínculos a otros sitios web}

Los sitios web presentes en internet constituyen un recurso comunicativo que pone a disposición del lector información relevante para aproximarse a los marcos de acción colectiva que facilitan los procesos de alineamiento de marcos, en la medida en que sus contenidos son evaluados por los interesados y actúan como esquemas de interpretación que habilitan a los individuos a 'localizar, percibir, identificar y etiquetar' los hechos de su espacio de vida y del mundo en general, de tal forma que los intereses, valores, creencias individuales y las actividades, objetivos e ideologías de las organizaciones del movimiento se tornen congruentes y complementarios (Snow et al. 1986).

Destacamos la conectividad que se pretende con el uso de internet que se refleja en que el $80 \%$ de los sitios existe la sección "Contacto", la cual permite vincular a los interesados con la organización, proyectar una futura participación o resolver dudas sobre los contenidos. Esto da cuenta de la importancia que los participantes le asignan a la web como medio de contacto y reclutamiento de los interesados en el movimiento.

La sección de vínculos a otros sitios web, permite visualizar las redes que constituyen el movimiento tanto a nivel local como global. Destacamos la presencia cada vez mayor de vínculos hacia redes sociales (web 2.0) de la organización, como es el caso de Facebook y YouTube. Secciones dedicadas a propósitos, proyectos, galería de imágenes, tienda virtual, foros, bibliotecas, buscadores, etc. reflejan la diversidad de temas que son tratados en los sitios.

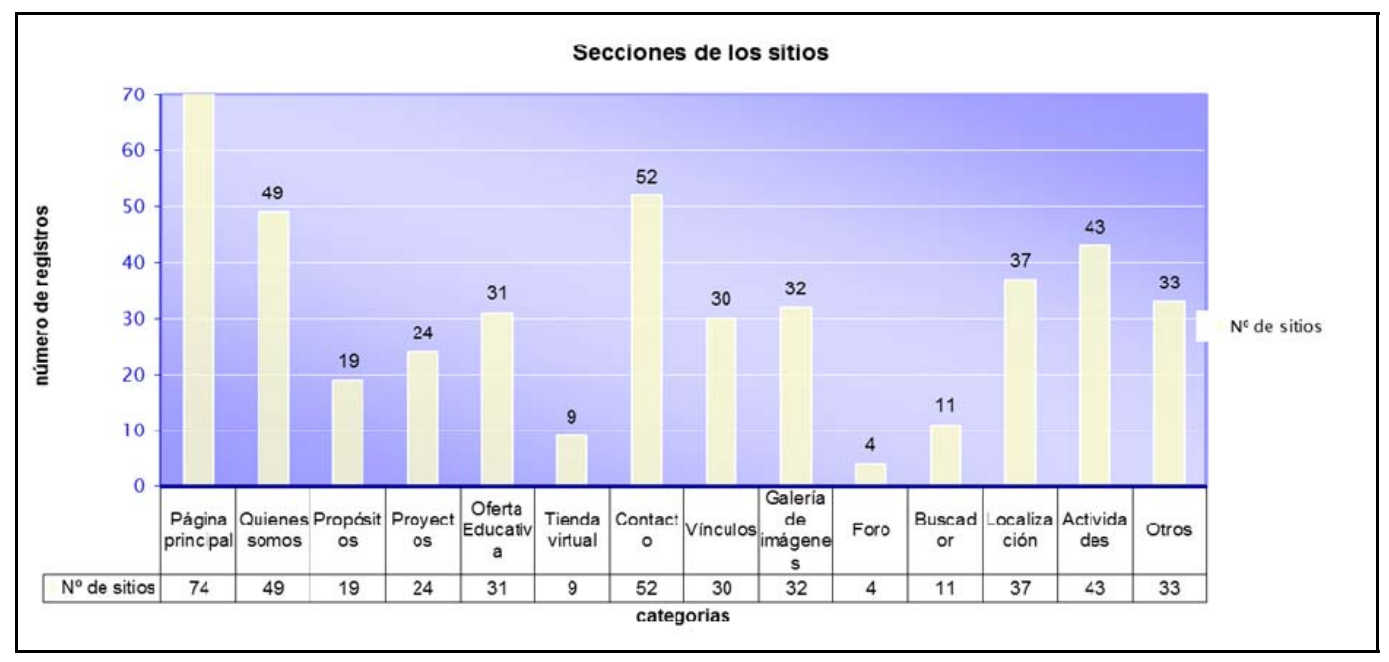

Figura 1. Secciones de los sitios web 
Las secciones referentes a la identidad y las actividades de la organización son fundamentales en la utilización de la web de parte de las organizaciones del movimiento.

Constatamos un énfasis en contactar a los interesados con las organizaciones, expresado en el hecho de que todos los sitios que utilizan foros, artículos y listas de correo, dan a conocer su dirección de correo electrónico.

Mostramos que los sitios que presentan foro corresponden a centros educativos, en los cuales el foro participativo a través de la web se utiliza con el fin de propiciar una comunidad de aprendizaje en torno a los diferentes tópicos que se trabajan al interior del movimiento. Con ello, se configura un espacio de colaboración, en el que la comunidad virtual facilita la participación y horizontalidad que ofrece este formato.

Gracias a internet las organizaciones del movimiento y sus participantes comparten espacios de convergencia y coordinación utilizando esta tecnología al servicio de la acción social transformadora.

La utilidad del uso de Internet varía en función del tipo de organización: en el caso de las ecoaldeas autónomas y centros educativos, sirve para promover actividades; en otros casos, cómo las redes de ecoaldeas y las revistas electrónicas, se utiliza como plataforma para la coordinación entre distintos proyectos, para la difusión de principios, acuerdos y experiencias de los participantes del movimiento.

Los procesos comunicativos se dan hacia el interior del movimiento y hacia su entorno. Hacia el interior del movimiento podemos observar formas de comunicación interpersonal, entre las redes de participantes y entre organizaciones del movimiento. Hacia el entorno podemos observar una comunicación abierta a quienes acceden a los productos comunicativos promovidos por el movimiento.

En cada uno de estos niveles se hace uso de diferentes medios y formas de comunicación; por ejemplo, en las relaciones interpersonales se intercambian distintos productos comunicativos como libros, películas, documentales, a través de los cuales se difunden las ideas y visiones del movimiento; en las reuniones de redes se comparten visiones, se unifica el lenguaje, se evalúan los procesos y se nivelan las expectativas entre los participantes.

Un importante espacio de comunicación se da en los encuentros, llamados o convergencias donde se reúnen los representantes de las organizaciones a nivel regional, nacional e internacional. En estos encuentros se generan acuerdos respecto a las orientaciones generales y los objetivos del trabajo en red, intercambiando conocimientos y experiencias.

En los encuentros de red se nivelan y regulan las interacciones entre los participantes y se comparten los principios que guían la acción colectiva y la participación en el movimiento. En el siguiente apartado analizamos estas dimensiones como procesos fundamentales de los procesos de alineamiento de marcos. 


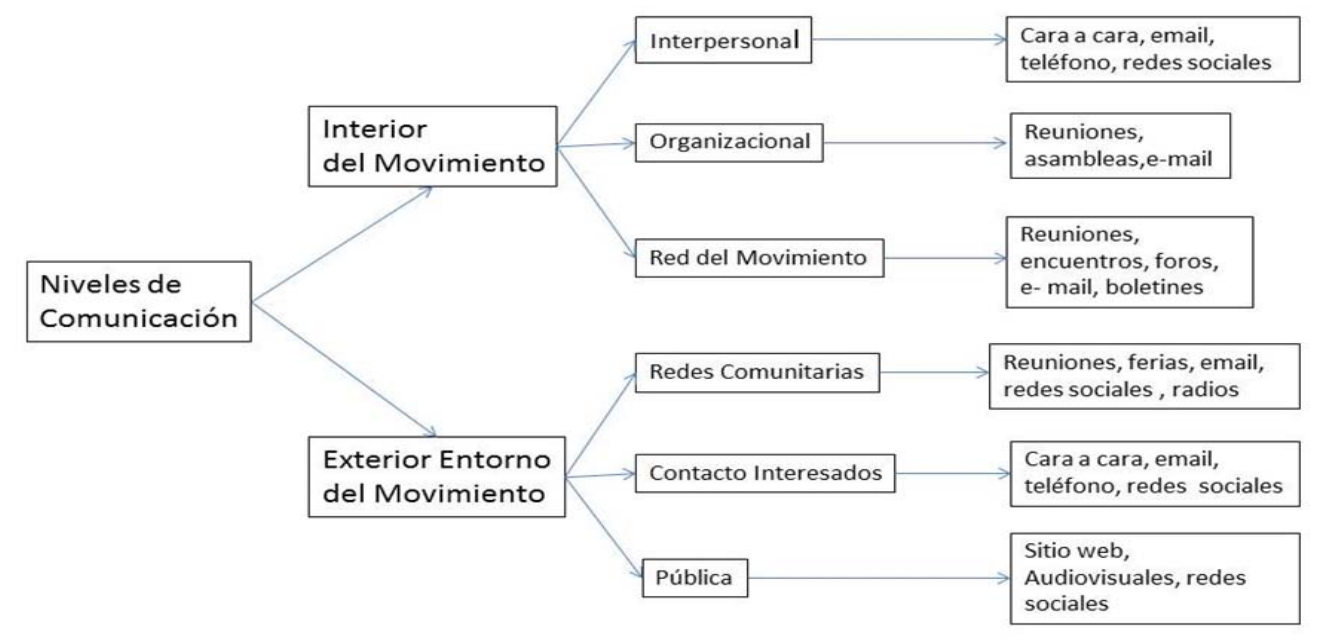

Figura 2. Niveles y formas de comunicación

\subsection{Análisis de los Marcos de Acción Colectiva}

Según Snow y Benford (1992), los 'marcos de acción colectiva' son los productos del proceso de enmarcar (framing) realizado por las organizaciones de movimientos sociales que están activamente implicadas en la producción de significados y en la lucha por los significados que deben prevalecer en la sociedad.

Para el análisis se consideran los resultados en función de los componentes de los marcos de acción colectiva (Gamson, 1992), los procesos de alineamientos de marcos (Snow y cols, 1986) y los marcos maestros (Snow y Benford, 1992).

\subsubsection{Componentes de los Marcos de Acción Colectiva}

A modo de integración, se analizaron los componentes de los marcos de acción colectiva según Gamson (1992), que corresponden a un sentido de injusticia, un sentido de agencia y un sentido de identidad.

En relación al componente injusticia, los participantes del movimiento cuestionan las bases de la sociedad de consumo contemporáneo, manifestando un descontento por el modelo capitalista que domina la política mundial, expresado en el sistema alimentario, la industrialización y el modelo de progreso en base al crecimiento ilimitado. 
El componente agencia lleva a los participantes a la búsqueda de soluciones y al trabajo en red, promoviendo los espacios de encuentro y coordinación que facilitan la cooperación y la ayuda mutua entre las organizaciones del movimiento. Los participantes optan por la construcción de alternativas y el desarrollo de emprendimientos que promueven la relocalización. Buscan el crecimiento del movimiento mediante la educación y la creación de más centros y proyectos que compartan las éticas y principios de la permacultura y el diseño de ecoaldeas.

El componente identidad se expresa en la definición de un nosotros y un ellos coexistiendo la identificación tanto con el ámbito local como con el global.

\subsubsection{Procesos de Alineamiento de Marcos}

Siguiendo la propuesta de Snow y cols, existen cuatro procesos de alineamiento de marcos entre los movimientos estudiados por los autores: 1) la conexión de marcos (frame bridging); 2) la amplificación de marcos (frame amplification); 3) la extensión de marcos (frame extension) y 4) la transformación de marcos (frame transformation).

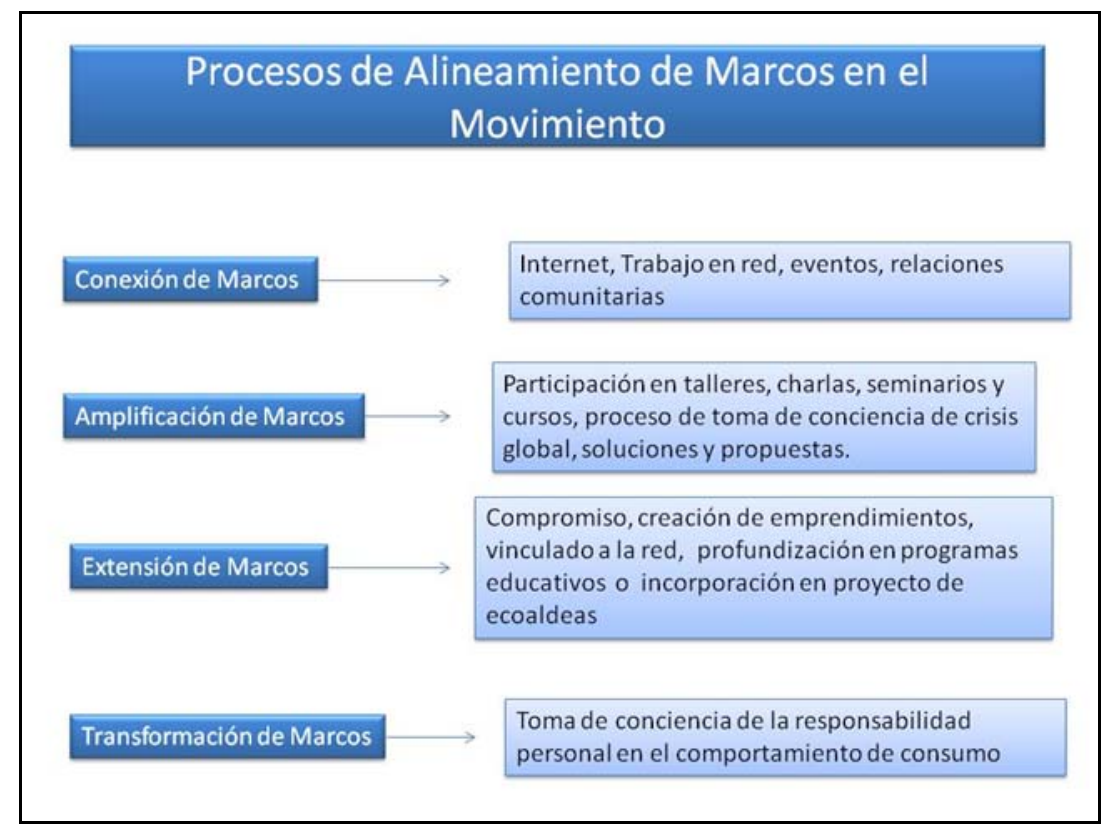

Figura 3. Procesos de alineamiento de marcos

1) La 'conexión de marcos' se refiere al enlace entre los marcos de una organización de movimiento social (OMS) y las creencias de grupos sociales que potencialmente simpatizarían con la ideología y objetivos promovidos por la organización pero que se encuentran 
estructuralmente desvinculados de la misma. Esta conexión se realiza principalmente mediante la difusión de información sobre los objetivos y la ideología de la organización de movimiento social (Snow y cols, 1986). La conexión de marcos se da fundamentalmente mediante las estrategias y espacios de trabajo en red. Estos espacios se dan tanto en encuentros presenciales (por ejemplo, en los eventos de las redes internas del movimiento), como en las redes comunitarias que se establecen con otras organizaciones y grupos territoriales, mediante ferias y seminarios. Internet permite la conexión de marcos, mediante la interacción, las redes sociales y los contenidos de los sitios web. Queda a disposición de los cibernautas los temas y propuestas que se ofrecen en los sitios web del movimiento.

2) La 'amplificación de marco' es la tarea de clarificar y fortalecer un marco interpretativo de una organización sobre un determinado problema para que se vincule con los marcos de los potenciales participantes. Esto se realiza identificando, idealizando o destacando valores supuestamente básicos para los potenciales participantes y acentuando creencias sobre la seriedad de los agravios, las causas del problema, los antagonistas y la eficacia de la acción colectiva. Los procesos de amplificación de marcos se pueden observar en la participación de talleres, charlas, seminarios y cursos. Los interesados comienzan su camino de aprendizaje experimentando un proceso de toma de conciencia de la evidencia de la crisis global para luego sumergirse en el abanico de soluciones y propuestas que se están desarrollando en los proyectos del movimiento.

3) Finalmente, el proceso de 'extensión de marco' consiste en extender la amplitud de un marco original del movimiento para alcanzar los principios, intereses y puntos de vista de los potenciales participantes que, aun no siendo de mucha importancia para el movimiento, son valorados por éstos. Esta tarea se realiza definiendo los objetivos o actividades del movimiento de modo que atienda o sea congruente con los intereses o valores de los potenciales participantes. La extensión de marco en el caso del movimiento se desarrolla cuando los participantes o interesados se comprometen con el movimiento, ya sea mediante la creación de algún emprendimiento vinculado a la red, la profundización en los programas educativos de especialización o en la incorporación a un proyecto de ecoaldeas. La participación activa en alguno de estos espacios abre un universo de conversaciones, redes, actividades, proyectos y experiencias en los que ya se está contribuyendo a la acción social con mayor nivel de compromiso y experimentación activa en los proyectos, soluciones y propuestas que van surgiendo en las organizaciones y redes del movimiento.

En estos tres procesos de 'alineamiento de marcos' los valores o creencias de los individuos son compatibles con los marcos interpretativos de los movimientos 
sociales. Pero cuando los valores o programas que los movimientos promueven no son compatibles con los marcos de los individuos a los que se quiere influenciar, se intenta la estrategia de alineamiento denominada 'transformación de marco'. La 'transformación de marco' comprende dos tipos de cambios. El primero es un cambio en la evaluación de una situación definida como tolerable y como producto de la mala suerte, hacia una situación ahora definida como intolerable, injusta o inmoral. El segundo se refiere al cambio en la atribución de culpa o responsabilidad por los agravios o problemas.

La transformación de marcos en el caso del movimiento se desencadena a partir de la toma de conciencia de la responsabilidad personal en el comportamiento de consumo para alimentar al sistema capitalista industrial que está generando daños muy graves en el ecosistema, que ponen en peligro la continuidad de la vida en la tierra tal como la conocemos. La transformación de marcos se ve fortalecida al ofrecer un abanico de posibilidades de realizar acciones concretas para el cambio de paradigma cultural que se ve como urgente y necesario frente a las evidencias de crisis civilizatoria que se comparten en el movimiento.

4.2.3. Los marcos propositivos para el cambio social: modelos de desarrollo para el futuro que se presentan como solución a las crisis sistémicas

La proyección de un paradigma de vida alternativo, sobre el que se construyen sueños compartidos y futuros posibles, orienta la construcción de objetivos y propósitos de las organizaciones del movimiento. En el caso del movimiento de ecoaldeas las prácticas sociales responden a propósitos compartidos, a propuestas de proyectos, programas educativos, ejercicios de diseño, estrategias de acción y prácticas transformadoras que configuran un marco de futuro o propositivo, compuesto tanto por las visiones más utópicas, como por proyectos y acciones concretas en las que se expresan las propuestas o modelos de desarrollo sostenible desde el movimiento.

Al analizar las propuestas de cambio social postulamos la existencia de marcos propositivos, que dan sentido a la participación y a la acción social con una visión de futuro compartida. Desde estos marcos se configuran modelos de desarrollo sostenible y prácticas comunitarias que incorporan la conciencia ecológica como una acción social transformadora y regenerativa que promueve cambios profundos al paradigma cultural.

Sobre la visión de futuro, las mismas ecoaldeas y centros demostrativos de permacultura son modelos de desarrollo para el futuro que se presentan como solución a las crisis sistémicas. Otras visiones plantean cambios graduales como el que promueve el movimiento de transición, cuya propuesta se centra en trabajar en conjunto con las autoridades locales por el desarrollo sostenible de los territorios, compartiendo tecnologías, metodologías, principios y aprendizajes. 


\subsubsection{Los Marcos Maestros en el Movimiento}

Los 'marcos maestros' son un tipo de marco de acción colectiva más general y elaborado que sería el que permite la agrupación por semejanza entre diversos movimientos sociales durante determinados ciclos de protesta y por el prolongamiento de estos ciclos históricos.

Los marcos 'maestros', subrayan Snow y Benford (1992), realizan las mismas funciones que los marcos de acción colectiva específicos de determinados movimientos; o sea, también son modos de puntualización, atribución y articulación, sólo que en una escala más amplia influenciando los marcos de acción colectiva de otros movimientos sociales. Los marcos maestros, por tanto, funcionan como modelos generales que inspiran marcos de acción colectiva específicos.

Uno de los marcos maestros del movimiento es el carácter antisistema, que permite compartir el descontento con el conjunto de movimientos sociales antisistema, antiglobalización y anticapitalista.

Otro marco maestro lo constituye el principio de sustentabilidad. Al amparo de una ética de la sostenibilidad es posible establecer puentes entre las propuesta del movimiento y las expresiones del movimiento ecologista, como también con las propuestas de desarrollo sostenibles, expresadas tanto en políticas públicas como en diversas organizaciones del mundo público y privado que han asumido el imperativo de la sustentabilidad y el cuidado del medio ambiente como componentes de sus políticas, valores compartidos y ejes estratégicos de desarrollo.

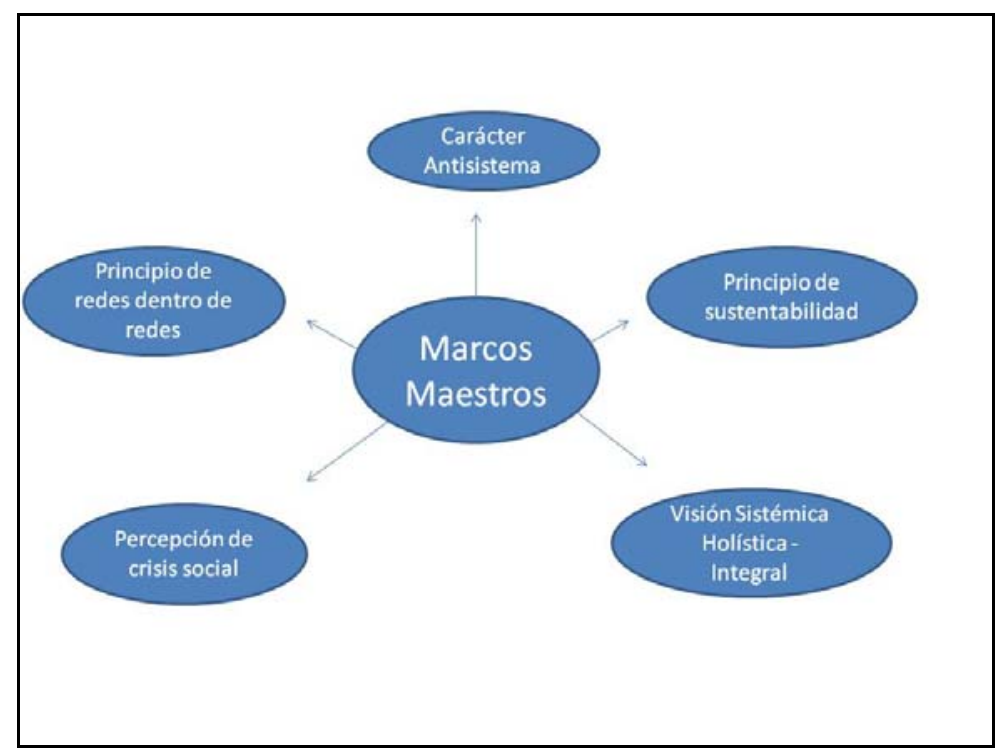

Figura 1. Marcos maestros de Acción Colectiva 
Siendo el pensamiento holístico o sistémico una de las competencias que buscan desarrollar las organizaciones del movimiento en sus participantes, podemos entender la observación de patrones y las relaciones sistémicas que se expresan en la naturaleza y que forman parte de la complejidad; se configuran también como un marco maestro que guía el diseño y comprensión, actuación e integración de los seres humanos en relación a sus territorios. Este marco permite establecer puentes con otros movimientos y sistemas de pensamiento que promueven una visión integral del ser humano y la naturaleza, lo cual contrasta con la visión moderna, lineal, modular y fragmentada del paradigma positivista racional sobre el que se funda nuestro orden social.

La percepción de crisis social es otro de los marcos maestros que son compartidos con otros movimientos sociales. El componente de injusticia de los marcos se expresa en el diagnóstico de la crisis sistémica y civilizatoria, que actúa como un llamado a la búsqueda de soluciones y a la acción colectiva transformadora.

Otro marco maestro que se puede identificar es la noción de red que actúa como un principio organizativo y estructural de la conformación del movimiento. El principio de redes dentro de redes permite que las fronteras o límites del movimiento sean difusas y como se propone en la conceptualización de los nuevos movimientos globales (Calle, 2007), están constituidos por discursos en red y globales que encadenan diversas dialécticas a la globalización como fuente de conflicto (capitalismo y exclusión social, género, interculturalidad, estructuras de poder no democráticas, relaciones con la naturaleza) generalmente desde ámbitos locales (ONG / redes locales o vecinales) o temáticos (deuda externa, inmigración, feminismo, etc.). Estas organizaciones constituyen redes horizontales caracterizadas por estructuras muy débiles o porosas que permiten la autonomía de los nodos locales y la interacción con distintos movimientos que comparten sentidos y significados.

\section{Conclusiones}

El estudio referido en este artículo abordó los procesos de mediación comunicativa y psicosociales que intervienen en los marcos de acción colectiva. Analizar la comunicación y los procesos de marcos permitió comprender e interpretar la naturaleza del movimiento y los sentidos sobre los que se fundamenta la acción colectiva y la cultura representada en los sitios web, construida en las redes y los espacios de interacción entre sus participantes y sus entornos.

Con esta investigación hemos comprobado que Internet se revela como un gran dispositivo para las mediaciones sociales, en general, y las mediaciones comunicativas, en particular. Un dispositivo de mediación social porque las páginas web son plataformas donde confluyen las inquietudes, necesidades, intereses, objetivos, propuestas, modelos de actuación, etc. de quienes las producen y las usan. Cada sitio web hace de espejo de un colectivo donde se miran los 
individuos y grupos que lo conforman y aquellos otros para los que representa un grupo de referencia (en la terminología de los sociólogos funcionalistas).

Internet es también un dispositivo de mediación comunicativa porque permite al movimiento social analizado, como a cualquier otro, construir y mostrar al mundo una representación de sí mismo y de su inserción en una o más redes. Con ello se consigue mediar en el conocimiento de sus integrantes y de otros ciudadanos que, sin serlo, toman conciencia de su existencia, pautas y actuaciones. A los miembros, les ofrece una identidad social, con la que afrontar los retos del presente de manera organizada; a los no miembros les ofrece la posibilidad de conocer sobre el entorno y sobre modos de vida alternativos y posibles; y, llegado el caso, la posibilidad de integrarse en un colectivo que, en cierto modo, ya les está mostrando cómo es, qué hace y a qué aspira.

\section{Referencias bibliográficas}

Calle, A. (2003). Los nuevos movimientos globales.Papeles del CEIC, $\mathrm{n}^{\circ}$ 7, CEIC (Centro de Estudio sobre la Identidad Colectiva), Universidad del País Vasco, Recuperado el 1 de Octubre desde http://www.ehu.es/CEIC/papeles/7.pdf.

Calle, A. (2007). La democracia (radical) a debate: los nuevos movimientos globales. MOVIMIENTOS SOCIALES Y ACCIÓN COLECTIVA. IX Congreso de Sociología, Barcelona. Recuperado el 5 de Septiembre desde http://www.dialnet.unirioja.es/descarga/articulo/2480857.pdf.

Gamson, W.A, (1992). The social psychology of collective action. en A. Morris y C. Mueller (eds), Frontiers in social movements theory, (pp. 53 - 76), Yale University Press: New Haven.

Hunt, S. Benford, R. y Snow, D. (1994). Marcos de Acción Colectiva y Campos de Identidad en la Construcción Social de los Movimientos en LARAÑA, E. y GUSFIELD, J., Los nuevos movimientos sociales: de la ideología a la identidad, Madrid:CIS.

Labbé, J. F. (2011). Movimiento estudiantil en chile: repertorios de acción, marcos de acción colectiva, impactos y desafíos para la política pública.

Circunstancia,11. Recuperado de http://www. ortegaygasset.edu/fog/ver/1534/circunstancia/anoxi---n--31---mayo-2013

Laraña, E. \& gusfield, J. (1994). Los nuevos movimientos sociales: de la ideología a la identidad. Madrid: CIS.

Maldonado, M. A. (2008). La globalización de los movimientos sociales y el orden liberal. Acción política, resistencia cívica, democracia. Revista Española de Investigaciones Sociológicas, 124(1), 11-44.

Martín Serrano, M. (2008). La Mediación Social, Madrid: Akal. 
Martín Serrano, M. (2004). La Producción Social de Comunicación, Madrid: Editorial Alianza.

Ortiz, R. R. (2008). Cibercultura: metáforas, prácticas sociales y colectivos en red. Revista Nómadas, 28, 8-20.

Pereira Salazar, C. A. (2015): Marcos de Acción Colectiva en los sitios web del Movimiento Global de Ecoaldeas. UCM, tesis doctoral.

Snow, D. y Benford, R. (1992). Master Frames and Cycles of Protest en A. Morris, y C. Mueller (Eds.) Frontiers in Social Movement Theory. (pp. 133 - 155). Londres: New Heaven Yale University Press.

Snow, D., Rochford, E., Worden, S. y Benford, R. (1986). Frame alignment process, micromobilization and movement participation. American Sociological Review, Vol 51, 464- 481.

Snow, D. y Benford, R. (1988). Ideology, Frame Resonance and Participant Mobilization en Klandermans, B., Kriesi, H. y Tarrow, S. (eds.), From Structure to Action, Greenwich: JAI Press.

Tarrow, S. (1997). Poder en movimiento. Los movimientos sociales, la acción colectiva y la política, Madrid: Alianza. 